Die energiepolitische Dimension der Erweiterung der Europäischen Union

\section{Unterschätzte Brisanz}

\section{Die Energiepolitik der EU wird durch die anstehende Osterweiterung stärker geprägt werden als manch anderer Politikbereich. Steigerung der Energieeffizi- enz, Umgang mit Kohle- und Atomkraftwerken sowie Klimapolitik sind nur die wichtigsten politischen Herausforderungen für Nachhaltigkeit in einer erwei- terten Union.}

$\mathrm{F}$ Von Felix Cbristian Matthes ür die Energiepolitik besteht keine spezifische EU-Kompetenz - und dies obwohl sich zwei der Gründungsverträge, der im Sommer 2002 ausgelaufene Vertrag über die Europäische Gemeinschaft für Kohle und Stahl sowie der Euratom-Vertrag, explizit auf den Energiesektor beziehen. Zudem wurde über Politikfelder mit weitreichenden EU-Kompetenzen, wie Binnenmarkt, Wettbewerb und Umwelt, der Energiesektor signifikant umgestaltet - hier sei nur auf die Liberalisierung der Strom- und Gasmärkte hingewiesen. In den Beitrittsverhandlungen hat sich dieser Zustand mehrfach als problematisch erwiesen. Trotzdem hat die anstehende Erweiterung eine erhebliche energiepolitische Dimension. Einige energiepolitische Debatten in der erweiterten EU zeichnen sich bereits ab, hat doch der Energiesektor in den meisten der Mitgliedsstaaten eine stärkere sozialpolitische (Energiepreise), industriepolitische (vor allem Kohle), aber auch geostrategische (Kernkraftwerke, Energieimporte) Brisanz als in den bisherigen Mitgliedsstaaten.

Der europäische Binnenmarkt für Energie wird sich mit der anstehenden Erweiterung erheblich vergrößern. Während die Bevölkerung der EU um ca. 20 Prozent zunimmt, steigt die Wirtschaftskraft der EU durch die Osterweiterung angesichts des bestehenden Wohlstandsgefälles jedoch nur um 4 Prozent! Angesichts der auch bis auf weiteres geringen Wirtschaftskraft der Beitrittsstaaten wird damit den wirtschaftlichen Folgen (auch) von Energie- und Umweltpolitik bzw. der Effizienz der ergriffenen Maßnahmen eine erhebliche Bedeutung zukommen.

In Bezug auf die Energiemärkte stehen die zukünftigen Mitgliedsstaaten dabei vor einer zweifachen Herausforderung. Sie müssen

1. innerhalb relativ kurzer Zeit die Energiepreise und die Energiemärkte liberalisieren und parallel dazu
2. auch aus umweltpolitischen Gründen in hohem Tempo die Sanierung und Erneuerung ihrer Anlagen vornehmen.

\section{- Herausforderungen Liberalisierung ...}

Die Restrukturierung des Energiesektors ist in den verschiedenen Staaten in unterschiedlichem Maße weit gediehen. Die derzeitigen Bestrebungen, die Energiemarktliberalisierung in der EU zu beschleunigen, stellen jedoch für die Beitrittsstaaten eine zusätzliche Herausforderung dar und erhöhen den Anpassungsdruck für die Energiewirtschaft massiv.

Eine wichtige Rolle bei der Restrukturierung des Energiesektors spielt die Privatisierung der Energieversorgungsunternehmen. In den bisher erbisher schon stärksten Unternehmen der EU, sodass die Privatisierungen in den EU-Beitrittsstaaten bereits zur weiteren Unternehmenskonzentration bzw. zur deutlichen Erhöhung der Marktmacht einzelner Energieversorger in der europäischen Energiewirtschaft beigetragen haben und eine Fortsetzung dieses Trends befürchtet werden muss. Hieraus ergeben sich sowohl wettbewerbsrechtliche als auch liberalisierungsseitige Herausforderungen im Rahmen der EU, beispielsweise die Entflechtung der verschiedenen Versorgungsstufen oder stärkere Regulierung.

\section{-... Steigerung der Energieeffizienz ...}

In vielen der Beitrittsstaaten liegt die Energieeffizienz deutlich unter dem Niveau der heutigen EUMitgliedsstaaten. Der Primärenergieverbrauch je Einwohner in der EU-15 ist zwar meist deutlich höher als in den Beitrittsstaaten, die Energieproduktivität - ausgedrückt als Primärenergieverbrauch je Einheit BIP auf Basis von Kaufkraftparitäten - ist jedoch in den meisten Beitrittsstaaten um etwa den Faktor 2 schlechter als im Mittel der folgten Privatisierungsverfahren dominierten die
EU-15. Die Erhöhung der Energieeffizienz in den Beitrittsstaaten entwickelt sich damit zu einem zentralen Handlungsfeld, insbesondere wenn in diesen Staaten zukünftig ein erhebliches Wirtschaftswachstum erwartet wird. Der Grad der makroökonomischen Stabilisierung in den Beitrittsstaaten wird dabei insbesondere eine große Rolle für die Schaffung stabiler Rahmenbedingungen für Investitionen spielen, auch und besonders im Bereich der Energieeffizienz. Angesichts der begrenzten Kaufkraft und der liberalisierungsbedingt vor allem im Bereich der Haushalte und Kleinverbraucher zunächst steigenden Energiepreise kommt einer Erhöhung der Energieeffizienz auch eine starke soziale Komponente zu, was in der bisherigen EU-Energiepolitik nur am Rande eine Rolle spielte.

Eine ähnliche Situation ergibt sich für die Schadstoffemissionen. Für die Leitschadstoffe Kohlendioxid und Schwefeldioxid ergibt sich für die meisten Beitrittsstaaten ein deutlich höherer spezifischer Ausstoß als für die EU-15. Mit dem EU-Beitritt müssen vor allem die klassischen Luftschadstoffemissionen erheblich zurückgeführt werden. Dies erfordert große Anstrengungen und Aufwendungen, teilweise sind die Luftreinhaltungsmaßnahmen jedoch schon relativ weit gediehen.

\section{- ... Kohlepolitik ...}

Mit Polen wird der heute größte Produzent von Steinkohle in Europa Mitglied der EU. Obwohl seit Ende der achtziger Jahre die Steinkohleproduktion um fast die Hälfte verringert wurde, lag die aktuelle polnische Steinkohleproduktion im Jahr 2000 mit ca. 100 Millionen Tonnen noch um mehr als den Faktor 2 über der deutschen Steinkohleproduktion und 18 Prozent über der gesamten Steinkohleproduktion in der EU. Aktuelle Projektionen gehen für das Jahr 2010 von einem Rückgang auf 83 Millionen bis 2010 und auf 68 Millionen Tonnen bis 2020 aus. Aber selbst diese relativ langsame Rückführung der - ebenso wie in Deutschland wenig wettbewerbsfähigen - Förderung wird umfangreiche Subventionen für die heute bereits mit ca. 4,3 Milliarden US Dollar verschuldete Kohleindustrie erfordern und zu nicht unerheblichen politischen Konflikten im nationalen Rahmen führen: Die Zahl der Beschäftigten ging bereits von 370.000 im Jahr 1990 auf 155.000 im Jahr 2000 zurïck. Eine von der Dynamik ähnliche Situation ergibt sich - wenn auch in geringerem Umfang - für die Steinkohleförderung in der Tschechischen Republik. Diese betrug 1999 noch 15 Millionen Tonnen und liegt damit etwa auf dem Niveau Spaniens. 
Unter den neuen EU-Mitgliedsstaaten befinden sich einige Länder mit Braunkohleförderung: Ungarn mit 14 Millionen Tonnen, aber auch einige Länder mit einer deutlich größeren Förderung von 40 bzw. 60 Millionen Tonnen Braunkohle (Tschechien bzw. Polen). Da gegenwärtig in der EU nur Deutschland (168 Millionen Tonnen), Griechenland (63 Millionen Tonnen) und - mit Abstrichen - Spanien (9 Millionen Tonnen) über eine nennenswerte Braunkohleförderung verfügen, wird sich die Rolle der Braunkohle in der Energiepolitik einer erweiterten EU verstärken, was vor allem im Kontext der Klimapolitik zu erheblichen Konflikten führen kann.

Daher steht die Kohlepolitik der EU mit dem Beitritt dieser beiden Länder vor einer neuen Situation, die nicht ohne Rückwirkungen bleiben wird.

\section{... und Atomenergie}

Im Energiemix der meisten Beitrittsstaaten spielt die Kernenergie zwar eine geringere Rolle als in der heutigen EU. Gerade weil die EU mit dem Euratom-Vertrag zwar über großzügige - und zunehmend umstrittene - Förderinstrumente für die Kernenergie verfügt, aber keine allgemein verbindlichen Sicherheitsstandards gelten, ist in den Beitrittsverhandlungen hilfsweise als Maßstab für die Forderung nach ,hohen Sicherheitsstandards im Nuklearbereich“ ein „Vergleich mit den in allen Mitgliedstaaten geltenden Praktiken und Regelungen" herangezogen worden. In den Beitrittsstaaten werden jedoch eine ganze Reihe von Reaktoren betrieben, die den ,in der EU gemeinhin geltenden Anforderungen und Praktiken" nach übereinstimmender Auffassung nicht genügen und (schwere) Auslegungsmängel aufweisen. Mit einigen Staaten wurde deshalb im Zuge des EU-Beitritts die Schließung von Reaktoren vereinbart, wobei die Umsetzung dieser Vereinbarung teilweise noch nicht gesichert ist. Aber auch die Sicherheitsstandards der anderen Reaktoren sind und bleiben auch vor dem Hintergrund der aufgetretenen Konstruktionsschwächen und Qualitätsmängel bei der Anlagenerrichtung - äußerst fragwürdig. Ein Spezifikum für den Nuklearsektor in den Staaten Mittel- und Osteuropas sowie der früheren Sowjetunion bilden schließlich die meist schwachen Kapazitäten der entsprechenden Aufsichtsbehörden sowie Probleme der Sicherheitskultur in Staaten mit tiefgreifenden gesellschaftlichen Verwerfungen. Auch die Entsorgungsfrage sowie die Bereitstellung der Mittel für den Rückbau von Kernkraftwerken ist in den meisten Beitrittsstaaten ungeklärt. Mit der Erweiterung wird die in den letzten Jahren stark ge- schrumpfte atomfreundliche Koalition in der EU gestärkt, gerade das von der Kommission Ende 2002 präsentierte „Nuclear Package“ liefert ein erstes Indiz für diese neue Situation.

\section{Klimapolitik in der erweiterten EU}

In der ersten Verpflichtungsperiode des KiotoProtokolls von 2008 bis 2012 können Emissionsreduktionen in den zukünftigen Mitgliedsstaaten der EU nicht ohne weiteres angerechnet werden. Gleichzeitig müssen die beitretenden mittel- und osteuropäischen Staaten in den ab 2005 geplanten EU-Emissionshandel für KohlendioxidEmissionen auf Anlagenebene voll einbezogen werden. Die damit verbundenen Aspekte wie entsprechende Minderungsvorgaben, die zusätzlichen Angebote an Emissionszertifikaten oder die Entschärfung der „Verlagerungsdiskussion“ sind jedoch in der Diskussion um die Einfuihrung des EU-Emissionshandelssystems bisher keineswegs ausreichend berücksichtigt worden. Auch die Inkompatibilität zwischen der EU-Lastenteilung zum Kioto-Protokoll - aus der die Minderungsvorgabe von 21 Prozent für Deutschland folgt - und dem EU-Emissionshandelssystems mit ihrem erweiterten Mitgliederbestand kann noch zu erheblichen Problemen führen. In jedem Fall werden vor diesem Hintergrund so genannte Joint Implementation-Projekte mit den neuen Mitgliedsstaaten eine wichtige Rolle spielen.

\section{Fazit}

Auch ohne Basis in den Verträgen werden durch die EU-Osterweiterung ,alte“ energiepolitische Themen neue Brisanz erhalten, deutliche Verschiebungen bei den Kräfte- und Interessenverhältnisse sind abzusehen. Energiepolitik auf EU-Ebene wird ohne Zweifel wichtiger. Schließlich erfordert die mehr als 1.000 Kilometer lange Grenze Polens zu Russland und Ukraine auch eine stärkere energieund umweltpolitische Komponente in den Außenbeziehungen der EU. Die laufenden Bemühungen um die Ratifikation des Kioto-Protokolls durch Russland sind hier die erste und bestimmt nicht die letzte Bewährungsprobe.

\section{Der Autor}

Dr. Felix Christian Matthes ist Koordinator des Bereichs Energie \& Klimaschutz im Öko-Institut. Kontakt: Öko-Institut, Novalisstraße 10, 10115 Berlin, E-Mail: matthes@oeko.de

\section{Neu im ökom Verlag}

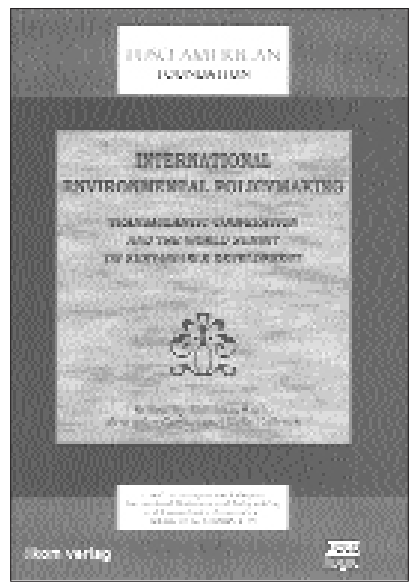

International Environmental Policymaking

Transatlantic Cooperation and the World Summit on Sustainable Development

Environmental policy-making, understood as collective and coordinated efforts to mitigate the impact of human activities on the natural environment, takes place on all levels of governance. The focus of this volume is on international and global environmental policy-making, with specific attention devoted to the role of transatlantic cooperation. The book documents the papers and discussion during an international expert workshop on the same topic, held at the Luso-American foundation in Lisbon in February 2000.

Matthias Buck was trained lawyer and political scientist and is an Associate Fellow with Ecologic.

Alexander Carius is co-founder and managing director of Adelphi Research.

Kelly Kollman is a Ph.D. in the Departement of Political Science at the George Washington University in Wahington D.C.

\section{www.oekom.de}

ed: Buck, Matthias; Carius, Alexander; Kollman, Kelly ökom Verlag, München. 2002; 369 pages; 23,40 Euro (plus porto); ISBN 3-928244-89-2

Erhältlich bei pan adress,

Semmelweisstraße 8, D-82152 Planegg, Fon $++49 /(0) 89 / 857$ 09-155, Fax -131, kontakt@oekom.de 
(c) 20I0 Authors; licensee IÖW and oekom verlag. This is an article distributed under the terms of the Creative Commons Attribution Non-Commercial No Derivates License (http://creativecommons.org/licenses/by-nc-nd/3.o/), which permits unrestricted use, distribution, and reproduction in any medium, provided the original work is properly cited. 